\title{
Validation of a Questionnaire to Diagnose the Baumann Skin Type in All Ethnicities and in Various Geographic Locations
}

\author{
Leslie Baumann ${ }^{1,2}$ \\ ${ }^{1}$ Baumann Cosmetic and Research Institute, Miami, USA \\ ${ }^{2}$ Division of Cosmetic Dermatology, University of Miami, Miami, USA \\ Email:DrB@derm.net
}

Received 3 November 2015; accepted 12 March 2016; published 15 March 2016

Copyright (C) 2016 by author and Scientific Research Publishing Inc.

This work is licensed under the Creative Commons Attribution International License (CC BY). http://creativecommons.org/licenses/by/4.0/

\section{Abstract}

The Baumann Skin Typing System diagnoses patients as having one of 16 skin types based on their answers to a validated questionnaire [i] known as the Baumann Skin Type Indicator [ii]. The BSTI questionnaire has been tested over the last decade on over 200,000 people of various ages and ethnicities in different geographic locations around the world. In this study, data were collected from 52,862 patients to compare skin type prevalence between those who presented to doctor's offices and those who took the quiz without supervision online. The most common skin types varied only slightly between patients that took the quiz online and those that completed the questionnaire in their doctor's office. This indicates that the prevalence of skin types seen in the doctor's office is similar to that in the general population and that supervision is not necessary to get an accurate result on the BSTI. [iii] In addition, comparison of data gathered in China, Korea, and the US did not show a significant difference in skin type prevalence between Asian and Caucasian skin types. [iv] This study demonstrates that the English version of the BSTI is valid for English speaking patients online, and in doctors' offices in the US, China and Korea.

\section{Keywords}

Skin Type, Regimen, Cosmeceutical, Skin Care, Oily, Dry, Sensitive, Pigmented, Wrinkled, Photoaged, Melasma, Pre-Operative, Post-Operative, Acne, Rosacea, Contact Dermatitis, Antioxidants, Retinoids, Baumann Skin Type, BSTI, Skin IQ, Skin Type Solutions, Systems-Based Approach, Standardized Methodology, Validated, Validation, Scientific, Ethnicity, Asian, Hispanic, Caucasian, Questionnaire, Diagnostic, Diagnose, Prescribe, Educate, Outcomes, Compliance 


\section{Introduction}

There is much misinformation about the difference in skin biology among various ethnicities. Although skin tone varies among various ethnicities, the underlying biology of the skin remains the same in all ethnicities with the exception of differences in skin color and the incidence of post -inflammatory hyperpigmentation. Skin color in darker-pigmented individuals occurs because melanocytes produce more melanin, the melanosomes are larger and more heavily melanized, and undergo degradation at a slower rate than in lighter skinned individuals [1]-[3]. Asians, Hispanics and African Americans suffer from the same skin issues such as an impaired barrier, tendency to inflammation and increased sebum production as other skin colors do. Darker types are at a lower risk for skin aging because the melanin provides a protective effect to the DNA. Rosacea may be less obvious in darker skin types, even when underlying redness is apparent. For this reason, we sought to determine if a questionnaire that was validated for use in Caucasian skin would be accurate for use in other ethnicities. The BSTI questionnaire was given to over 52,000 people and the results were compared by ethnicity. Dermatologists in Korea and China gave the questionnaire to their patients and the data were compared to results gathered online in the United States and in dermatologist's offices in the Unites States. The physicians in the USA, China and Korea felt that the Baumann Skin Type Indicator Questionnaire accurately reflected the dermatologic skin issues of their patients and was a useful diagnostic tool in their medical practice.

\section{Need for a Diagnostic Questionnaire That Is Accurate for All Ethnicities}

As skin care science has progressed and many new ingredients have been discovered, the efficacy of skin care products has improved as shown in multiple studies. However, a research setting does not mimic real life. In a research study, the investigators take great care in screening and choosing recipients most likely to experience improvement from the study product. In fact, detailed inclusion and exclusion criteria are used to determine who will be enrolled in a study. In the real world, patients self-select what skin care products they use based on celebrity endorsements, a friend's recommendation or product marketing with little regard for their underlying skin biology. Without a clear diagnostic process, it is very difficult to match the correct skin care products to the corrects in type. Using a photograph or a quick visual analysis is not enough information to determine skin type because that only shows the skin at a particular point in time and does not give any historical information. Historical information is crucial in determining skin type because it is important to understand how the skin reacts in various environments and when exposed to various types of products and situations. A validated method of skin type diagnosis is also important because patients notoriously are incorrect about their skin type as illustrated in a study of 94 Korean women who subjectively rated their skin type as oily, dry, or normal. The subjective self-rating was compared to sebometer measurements, which showed that subjects underestimated their skin's sebum production and often called themselves "dry" when in fact the skin was "oily” [4]. Seasonal variations in sebum secretion can confuse subjects [5] as can any biases or myths that they believe about their skin.

Asking patients questions about their skin is also a form of patient education. When patients are asked to check off a series of behavioral habits such as smoking, use of tanning beds, consumption of fruits and vegetables, how many hours of nightly sleep, and other lifestyle choices or patterns, they begin to consider that these habits may have an effect on their skin health. This helps open the dialogue and expands communication between the patient and physician. The process of “diagnose, prescribe and educate" is a strategy used to initiate skin care discussions between the patient and the physician. A questionnaire facilitates all three of these steps. In fact, diagnosing the patient's skin type paves the way for distributing customized patient education material directly to those for whom the information is most applicable.

Using a validated questionnaire to select patients for a treatment method is a similar approach to using inclusion and exclusion criteria in a research study and allows a standardized methodology to track product efficacy according to skin phenotype. This systems-based approach sets the stage for physicians to systematically track efficacy and collaborate with colleagues to discuss and publish results and address complications arising from skin care recommendations including topical prescription medications.

\section{The Baumann Skin Type Indicator}

The Baumann Skin Type Indicator (BSTI) is a validated questionnaire [6], which consists of a 3- to 5-minute computer-based questionnaire assessing 4 main parameters of the skin: 1) oily vs. dry, 2) sensitive vs. resistant, 3) pigmented vs. non-pigmented, and 4) wrinkle-prone vs. tight. Depending on the way the questions are ans- 
wered, the patient is diagnosed as having a distinct skin phenotype known as the Baumann Skin Type ${ }^{\circledR}$. There are 16 possible Baumann Skin Types based on these four parameters. The Baumann Skin Type is designated by 4 letters and a corresponding color and number (Figure 1). For example, a dry, resistant, non-pigmented, wrinkle-prone type would be designated as DRNW. The Baumann Skin Types may also be designated by a scalloped colored octagon containing a number corresponding with the skin type (Figure 2). The Baumann Skin Type (BST) can vary with seasons, hormone fluctuation, lifestyle changes, pregnancy, medications, move to a new geographic location, and other factors. Patients should retake the questionnaire if they have experienced any of these changes so that their skin care regimen can be properly adjusted. The goal of skin care targeted to skin type is to change the skin type to the most desirable skin type: ORNT (hydrated, non-inflamed, even skin tone, no wrinkles.)

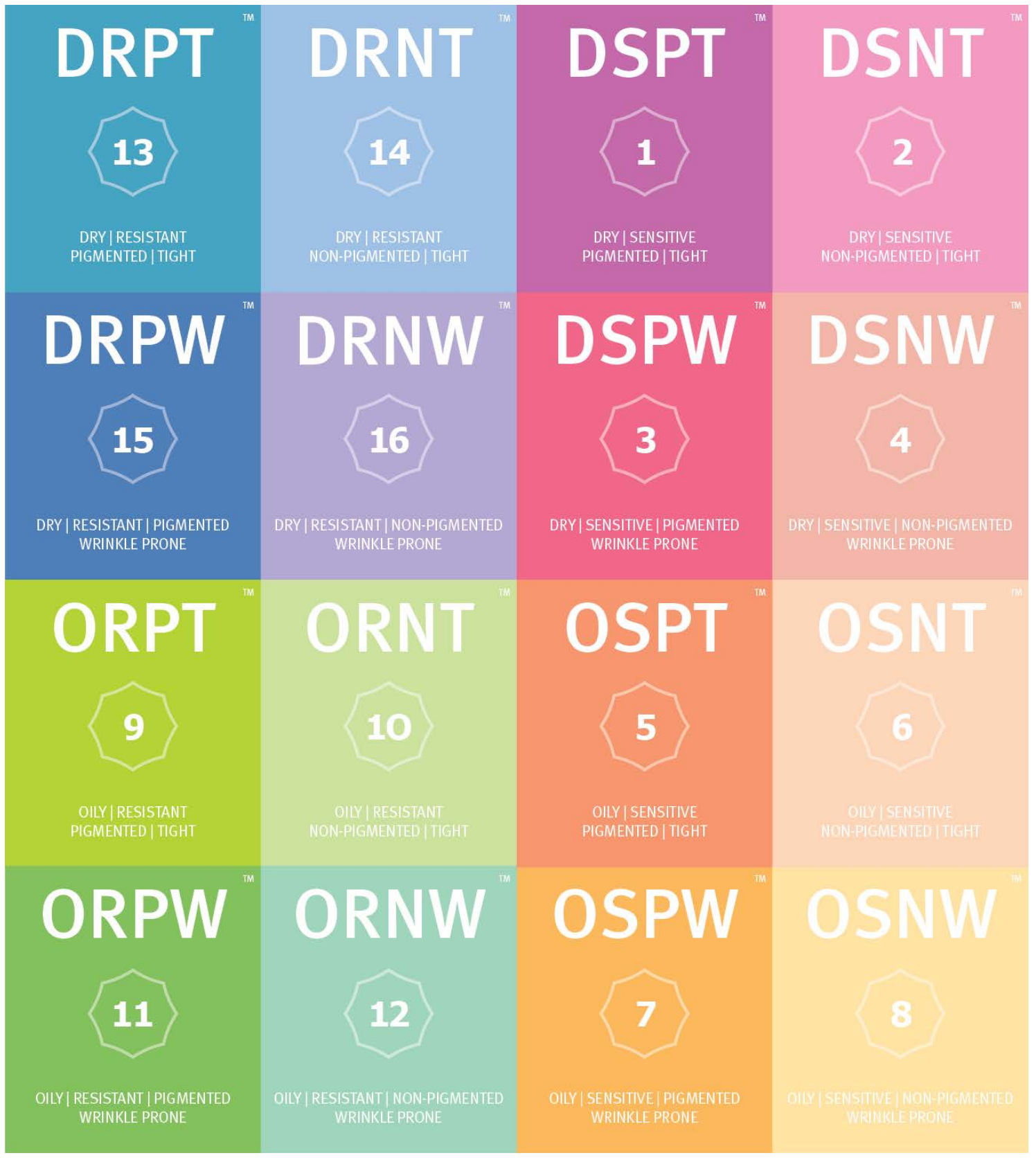

This bingo card design of the 16 Baumann Skin Types is a trademark of Metabeauty, Inc. and is used with permission.

Figure 1. The 16 Baumann skin types. 


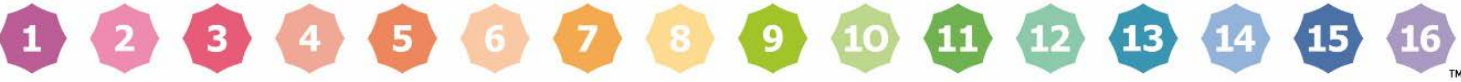

This scalloped octagon design of the 16 Baumann Skin Types is a trademark of Metabeauty, Inc. and is used with permission.

Figure 2. The 16 Baumann Skin Types are designated by scalloped octagons with a corresponding number and color.

\section{Characteristics of the 4 Parameters}

Dry (D) skin is characterized by an impaired lipid barrier surrounding the keratinocytes. An impaired barrier increases transepidermal water loss (TEWL) and renders the skin more susceptible to irritation from retinoids, acne medications, and other irritating ingredients. Having dry skin does not equate to having sensitive skin, because having dry skin alone is not enough to activate inflammatory cascades, which is necessary in order for inflammation to occur. However, dry skin types are more susceptible to the allergic type of sensitive skin because the impaired barrier facilitates entry of allergens.

Oily (O) skin is characterized by adequate or elevated sebum production. Sebum is a lipid-filled occlusive moisturizing substance that coats the surface of the skin. The occlusion caused by sebum leads to prevention of water evaporation (decreased TEWL) and entry of irritants into the skin. In other words, increased sebum production can mask an underlying defect in the skin barrier. For this reason, combination skin does not exist in the Baumann Skin Typing System. If the skin barrier is intact and the skin has a normal level of sebum function, the skin is considered an oily skin type. If the skin barrier is not intact but the skin produces enough oil to provide occlusion sufficient to prevent TEWL, then the skin is considered an oily skin type. If the skin does not have enough sebum to prevent water evaporation and the skin barrier is impaired, the skin is a dry type.

Sensitive (S) skin can be subdivided into 4 subtypes, each characterized by inflammation. The 4 sensitive skin types are: acne (pimples), rosacea (redness), burning/stinging, or allergic. Patients often exhibit several types of sensitive skin. For instance, a patient who has frequent rashes to skin care and develops pimples would have the acne, allergic subtypes of sensitive skin. It is important to distinguish the type of sensitive skin because they each require different kinds of cosmeceutical ingredients. The BSTI questionnaire identifies which sensitive skin subtypes the patient has by adding a number to the end of the 4 letters. For example, an oily, sensitive (acne), pigmented, wrinkle-prone patient would be designated OSPW1, while an OSPW type with rosacea would be designated OSPW2. For simplicity, these could also be designated as OSPW Acne Type or OSPW Rosacea Type. In some cases, the patient may have more than one type of sensitivity. For example, an OSPW who exhibits both acne and rosacea would be designated as an OSPW12 or as an OSPW Acne and Rosacea Type.

Resistant (R) skin types rarely suffer the four forms of inflammation described above and can tolerate a larger variety of products and procedures. They often require stronger products and procedures to see significant results.

Pigmented (P) skin types in the Baumann Skin Typing System have uneven skin tone due to overactive melanocytes. The "P" designation is not an indicator of ethnicity, but rather is an indicator that the skin needs lightening or brightening agents to even the skin tone. In the Baumann Skin Typing System, the pigmented skin types have melasma, postinflammatory hyperpigmentation, or solar lentigos.

Non-pigmented (N) skin types in the Baumann Skin Typing System have an even skin tone and do not require skin-lightening ingredients. A dark-skinned individual with even skin tone would be designated as an " $\mathrm{N}$ " skin type. A patient with freckles that they do not want to remove would be designated a "N" type because skin lightening is not desired. For this reason, the $\mathrm{N}$ vs $\mathrm{P}$ designation is both objective and subjective and depends on the patient's desired outcome.

Wrinkle-prone (W) skin types have lifestyle habits such as cigarette smoking, sun exposure, and tanning bed use that increase their risk for skin aging. Wrinkle-prone types should use aging prevention strategies that include daily sunscreen, retinoids, and antioxidants including ascorbic acid. Identifying these risk factors at a younger age will help begin prevention strategies sooner, which may help preserve the skin's strength, thickness and elasticity.

Tight (T) skin types are usually below 25 years old and do not have a history of sun exposure, smoking, and other lifestyle factors that make them more susceptible to skin aging. Although this skin type does not require antiaging ingredients, daily SPF is always recommended to minimize risk factors for skin aging and skin cancer. Teenagers with acne or young people with eczema fall into the T category. 


\section{Using the 16 Facial Skin Phenotypes to Prescribe a Skin Care Regimen}

Combination of the four parameters described above results in 16 skin phenotypes known as the "Baumann Skin Types ${ }^{\circledR}$," which are designated by four letters and a corresponding color and number (Figure 1 and Figure 2). Using this nomenclature allows physicians to use a standardized methodology that takes into account all four of the skin parameters, each of which contributes to the patient's skin phenotype and needs. Using this skin typing system is preferable to a disease-based nomenclature as illustrated by this example of a DSPW patient:

A patient with dry, sensitive, pigmented, and wrinkle-prone skin presents to the dermatologist. Normal standard of care would lead to a diagnosis as an "acne patient" and treatment with a combination of antibiotics, benzoyl peroxide, and retinoids. Antibiotics and benzoyl peroxide decrease the bacterial levels of Propionibacterium acnes while retinoids affect Toll-like 2 receptors and promote desquamation of retained keratinocytes. Using antibiotics, retinoids, and benzoyl peroxide is the standard of care for acne patients. However, the DSPT patient will likely respond poorly to this routine acne regimen. The defect in the skin barrier will allow increased penetration of the irritating benzoyl peroxide and retinoids resulting in dryness, inflammation, and scaling that may lead to postinflammatory hyperpigmentation. This poor response can lead to noncompliance with the acne medications and a poor outcome in which the patient is worse off than at the outset because now they have acne, dermatitis, and postinflammatory hyperpigmentation.

What went wrong? By diagnosing this individual as an "acne patient," the increased tendency of postinflammatory hyperpigmentation, inflammation, propensity to wrinkle and the impaired skin barrier went unconsidered and undiagnosed. It is routine for doctors-and promoted by insurance regulations-to assign a single diagnosis to a patient instead of combining diagnoses. In this case, the patient suffered from three underlying issues: risk of dyschromia, xerosis, and acne, which are considered discrete or independent phenomena. The patient would have an improved outcome if these three disorders were considered together as part of a distinct skin type known as DSPW 1 (Acne type).

The DSPW patient has an impaired skin barrier, which allows increased penetration of ingredients into the dermis due to a decrease of ceramides, fatty acids, and cholesterol in the lipid bilayer surrounding the keratinocytes. The impaired skin barrier seen in the " $D$ " skin type would permit increased penetration of ingredients such as benzoyl peroxide and topical retinoids, which yields an increase in the incidence of adverse effects from these ingredients (redness, peeling, skin stinging, and burning). The irritation from both benzoyl peroxide and retinoids is known to be dose dependent [7] [8]. Retinoids promote inflammation through several mechanisms, one of which impacts cytokine expression [9]. "S" skin types are most susceptible to develop inflammation at a certain dose of medication because of increased activity of internal pro-inflammatory factors such as neutrophil attracting cytokines [10]. This leaves them more vulnerable to inflammation from topically applied products in a dose-dependent manner. Combining the skin types " $D$ " and " $S$ " results in an exaggerated inflammatory response to an increased dose of a chemical agent, greatly increasing the likelihood of an adverse event. Because this patient also has a " $P$ " designation, his melanocytes have increased tyrosinase activity resulting in elevated production of melanin by melanocytes. In other words, he will develop hyperpigmentation after inflammation due to activation of melanocytes [11]. The fact that this patient is wrinkle prone should also be considered when prescribing a skin care regimen, so addition of antioxidants and sunscreen is crucial to counteract the pro-oxidant effects of benzoyl peroxide.

Taking all four of the parameters into consideration when prescribing a skin care regimen will improve outcomes because all of the important variables are taken into account. In addition, giving clear instructions to patients helps increase compliance, which also improves clinical outcomes and skin health.

\section{Incidence of the Individual Baumann Skin Types}

Over the last 10 years, the BSTI has been used to gather skin type data from around the world. It has been shown that the Baumann Skin Types are not equally distributed; some are much more common than others. In data collected from 52,862 patients (in doctors' offices and online to include people that have sought medical care and people who have not sought medical care), it was found that $65 \%$ of people were oily skin types while 35\% were dry types (Table 1). The most common skin types varied only slightly between patients that took the quiz online and those that completed the questionnaire in their doctor's office. This indicates that the prevalence of skin types seen in the doctor's office is similar to that in the general population [12]. In addition, comparison 
of data gathered in China, Korea, and the US (Table 2 and Table 3) did not show a significant difference in oily vs dry skin type prevalence between Asian and Caucasian skin types [13]. Although skin lightening products sell more in Asia than in the US, Table 4 and Table 5 show that the incidence of pigmented (uneven skin tone) types is not very different between Asians and other ethnicities.

Table 1. Combined data from physicians' offices (both dermatology and plastic surgery) and from the website SkinTypeSolutions.com show that oily skin types were more common than dry types.

\begin{tabular}{ccc}
\hline Baumann & Count & \% to total \\
\cline { 1 - 1 } Skin type & & $65 \%$ \\
\hline Oily & $\mathbf{3 4 , 2 0 3}$ & $35 \%$ \\
Dry & $\mathbf{1 8 , 6 5 9}$ & \\
Total & $\mathbf{5 2 , 8 6 2}$ & \\
\hline
\end{tabular}

Table 2. Data from physician's offices in US in 2006.

\begin{tabular}{ccc}
\hline Skin type & Count & \% to total \\
\hline Dry & 413 & $49 \%$ \\
Oily & 431 & $51 \%$ \\
Total & 844 & \\
\hline
\end{tabular}

Table 3. Data from physicians' offices in China and Korea in 2006 ("Unknown” did not complete the questionnaire).

\begin{tabular}{ccc}
\hline Baumannskin type & Count & \% to total \\
\hline Dry & 164 & $57.7 \%$ \\
Oily & 116 & $40.8 \%$ \\
Unknown & 4 & $1.41 \%$ \\
Total & 284 & $99.9 \%$ \\
\hline
\end{tabular}

Table 4. 39\% of Asians in China and Korea reported an uneven skintone.

\begin{tabular}{|ccc|}
\hline \multicolumn{2}{c}{ Asians } & \\
\hline Baumann & Count & \% to total \\
\hline Skin type & & $39 \%$ \\
\hline Pigmented & $\mathbf{1 1 0}$ & $60 \%$ \\
Nonpigmented & $\mathbf{1 7 0}$ & \\
\hline & $\mathbf{2 8 0}$ & \\
\hline
\end{tabular}

Table 5. All ethnicities combines reported 53\% unevenness in skin tone.

\begin{tabular}{|ccc|}
\hline & \multicolumn{2}{c}{ All ethnicities } \\
\hline Baumann & Count & \% to total \\
\hline Skin type & & \\
\hline Pigmented & $\mathbf{2 7 , 9 2 5}$ & $53 \%$ \\
Nonpigmented & $\mathbf{2 4 , 9 3 7}$ & $47 \%$ \\
& $\mathbf{5 2 , 8 6 2}$ & \\
\hline
\end{tabular}




\section{Conclusion}

The Baumann Skin Typing System assigns patients to one of 16 skin types based on their answers to a validated questionnaire [6] known as the Baumann Skin Type Indicator [14]. The questionnaire gives similar results among all ethnicities. Using the Baumann Skin Type nomenclature improves communication between physicians, scientists, researchers, aestheticians, and patients about skin care regimen efficacy and adverse events. Because studies have demonstrated that patients cannot properly self-diagnose their skin type, the Baumann Skin Type should be diagnosed using a validated questionnaire for that purpose [15]. The incidence of various skin type diagnoses is similar among Americans, Koreans and Chinese using this diagnostic tool, indicating that this tool is valid for English speaking patients in these 3 countries. The English version of the Baumann Skin Type Indicator has been shown to be valid for English speaking patients online, and in doctors' offices in the US, China and Korea. It is unknown at this time if the questionnaire is valid in other translations.

\section{References}

[1] Szabo, G., Gerald, A.B., Pathak, M.A., et al. (1969) Racial Differences in the Fate of Melanosomes in Human Epidermis. Nature, 222, 1081. http://dx.doi.org/10.1038/2221081a0

[2] Baumann, L. (2009) Cosmetic Dermatology. Chapter 13, Skin Pigmentation and Pigmentation Disorders, McGraw Hill, 98-109.

[3] Bolognia, J.L. and Pawelek, J.M. (1988) Biology of Hypopigmentation. Journal of the American Academy of Dermatology, 19, 217-255. http://dx.doi.org/10.1016/S0190-9622(88)70168-1

[4] Youn, S.W., Kim, S.J., Hwang, I.A., et al. (2002) Evaluation of Facial Skin Type by Sebum Secretion: Discrepancies between Subjective Descriptions and Sebum Secretion. Skin Research and Technology, 8, 168-172. http://dx.doi.org/10.1034/j.1600-0846.2002.10320.X

[5] Youn, S.W., Na, J.I., Choi, S.Y., et al. (2005) Regional and Seasonal Variations in Facial Sebum Secretions: A Proposal for the Definition of Combination Skin Type. Skin Research and Technology, 11, 189-195. http://dx.doi.org/10.1111/j.1600-0846.2005.00119.x

[6] Baumann, L., Penfield, R., Clarke, J. and Duque, D. (2014) A Validated Questionnaire for Quantifying Skin Oiliness. Journal of Cosmetics, Dermatological Sciences and Applications, 4, 78-84. http://dx.doi.org/10.4236/jcdsa.2014.42012

[7] Bucks, D., Sarpotdar, P., Yu, K., et al. (2009) The Development and Optimization of a Fixed Combination of Clindamycin and Benzoyl Peroxide Aqueous Gel. Journal of Drugs in Dermatology, 8, 634-638.

[8] Fluhr, J.W. (2014) Benzoyl Peroxide, in Pathogenesis and Treatment of Acne and Rosacea. Springer, Berlin Heidelberg, 419-423. http://dx.doi.org/10.1007/978-3-540-69375-8_56

[9] Babina, M., Guhl, S., Motakis, E., et al. (2015) Retinoic Acid Potentiates Inflammatory Cytokines in Human Mast Cells: Identification of Mast Cells as Prominent Constituents of the Skin Retinoid Network. Molecular and Cellular Endocrinology, 406, 49-59. http://dx.doi.org/10.1016/j.mce.2015.02.019

[10] Pasparakis, M., Haase, I. and Nestle, F.O. (2014) Mechanisms Regulating Skin Immunity and Inflammation. Nature Reviews Immunology, 14, 289-301. http://dx.doi.org/10.1038/nri3646

[11] Davis, E. and Callender, V.D. (2010) Postinflammatory Hyperpigmentation: A Review of the Epidemiology, Clinical Features, and Treatment Options in Skin of Color. Journal of Clinical and Aesthetic Dermatology, 3, 20-31.

[12] (2015) Data on File at Baumann Cosmetic and Research Institute, Miami, FL, October.

[13] (2006) Data on File at Baumann Cosmetic and Research Institute, Miami, FL.

[14] Baumann, L. (2009) The Baumann Skin-Type Indicator: A Novel Approach to Understanding Skin Type. In: Barel, A.O., Paye, M. and Maibach, H.I., Eds., Handbook of Cosmetic Science and Technology, 3rd Edition, Informa Healthcare, New York, 29-40. http://dx.doi.org/10.1201/b15273-5

[15] Baumann, L. (2010) The Baumann Skin Typing System. In: Farage, M.A., Miller, K.W. and Maibach, H.I., Eds., Textbook of Aging Skin, Springer, New York, 929-944. http://dx.doi.org/10.1007/978-3-540-89656-2_88 